\section{The interpretation of genetic tests in inherited cardiovascular diseases}

\author{
Lorenzo Monserrat, ${ }^{1}$ Andrea Mazzanti, ${ }^{1}$ \\ Martín Ortiz-Genga, ${ }^{1}$ \\ Roberto Barriales-Villa, ${ }^{1}$ \\ Diego Garcia-Giustiniani, ${ }^{1}$ Juan Ramon \\ Gimeno-Blanes ${ }^{2}$ \\ ${ }^{1}$ Complejo Hospitalario Universitario de A \\ Coruña, Insituto de Investigación \\ Biomédica de A Coruña, A Coruña; \\ ${ }^{2}$ Hospital Universitario Virgen de la \\ Arrixaca, Murcia, Spain
}

\section{Abstract}

The inherited cardiovascular diseases, including cardiomyopathies, channelopaties and inherited diseases of the aorta are heterogeneous conditions with highly variable morphologic and functional features, clinical presentation, evolution and prognosis. Hundreds of mutations in different genes have been associated with each one of these entities and it is likely that this genetic heterogeneity is one of the main reasons for the variability in their clinical expression. Information from the genetic studies may help the clinicians to diagnose the diseases in early stages, to identify relatives at risk and those who do not require periodic follow up, and may also provide prognostic information. An appropriate and accurate interpretation of the genetic tests is required to get all the potential advantages of these studies. This interpretation is not simple and requires information, specialized knowledge and dedication to the task. The first step is to decide which the appropriate genetic test is. Negative results do not exclude the disease and in that situation we need to decide whether to continue the screening or not. When the genetic study identifies one or multiple genetic variants we will have to evaluate their frequency in the general population (polymorphisms vs. mutations) and their pathogenicity. To establish whether a given variant is associated with the disease we have to integrate both basic and clinical information. When a variant is considered potentially pathogenic we still have to evaluate whether this variant explains the phenotype of the patient and of his/her family (more than one mutation may be present). Finally, we have to analyse all the available information about the consequences of the identified mutations and to integrate this information with all the available clinical data of the patient and family. With this approach, genetic test becomes a very useful tool in the management of all the inherited cardiovascular diseases.

\section{Introduction}

In the last 20 years our knowledge about the genetic causes of inherited cardiovascular diseases has experienced a great evolution. In the early 90 s, one mutation in the beta myosin heavy chain gene was associated with hypertrophic cardiomyopathy. ${ }^{1}$ Researchers thought that the cause of one of the most common familial cardiomyopathies had been discovered, but we quickly learnt that multiple mutations in the same gene were associated with the disease and that the MYH7 gene would only explain a certain number of cases. ${ }^{2}$ The advance in the genotyping technologies and the dedication of multiple research groups have progressively unveiled the implication of hundreds of different genes and thousands of mutations in the origin of the diverse inherited cardiovascular diseases. As an example, the first description of a mutation associated with long QT syndrome appeared in $1995 .^{3}$ The transition from research to clinical application is always a difficult process, and genetic testing has not been an exception to this rule. ${ }^{4}$ During a long time, the main limitation has been the high cost and low efficiency of the genotyping technologies. The technologic evolution will never stop, providing continuous improvements, but we already have reliable and cost-effective genotyping tools to support the routine application of genetics in the study of inherited cardiovascular diseases. ${ }^{5-7}$ However, the application of genetic studies in clinical practice requires not only the identification of the genetic variants (i.e. reading of the genetic code), but also an adequate interpretation of these variants (the comprehension of the message). This latter task is in fact becoming the most challenging problem with the arrival of new technologies. ${ }^{8}$

\section{Inherited cardiovascular diseases: a clinically relevant concept}

With the term inherited cardiovascular diseases we refer to a group of entities which are characterized by a familial presentation and are caused by rare genetic variants (mutations) with high or relatively high penetrance; being penetrance the proportion of individuals carrying a genetic variant that also express a related phenotype at a given age. The inherited cardiovascular diseases are usually considered monogenic disorders (one single genetic variant is responsible for the disease), even though a substantial proportion of the affected patients carries more than one pathogenic variant. We include in this group the cardiomyopathies (hypertrophic, dilated, restrictive, arrhythmogenic and non-compacted), the cardiac channelopathies (long and short QT, Brugada syndrome, catecholaminergic polymorphic ventricular tachycardia, inherited
Correspondence: Lorenzo Monserrat, Servicio Cardiologia Complejo Hospitalario Universitario A Coruña, As Xubias 84, A Coruña 15006, Spain. Tel: +34.981.611321 - Fax: +34.981.611321.

E-mail: lorenzo.monserrat.iglesias@sergas.es

Key words: genetics, cardiomyopathies, channelopaties, cardiovascular diseases.

Acknowledgements: this work was supported by grants of the Instituto de Salud Carlos III (FIS project PI081834), and the Spanish Cardiovascular Research Network RECAVA.

Contributions: the authors contributed equally. Conflicts of interests: LM, stock holder and consulting fees/honoraria from Health in Code SL; $\mathrm{AM}, \mathrm{MO}$, and DG, consulting fees from Health in Code SL.

Received for publication: 20 June 2011. Accepted for publication: 12 July 2011.

This work is licensed under a Creative Commons Attribution NonCommercial 3.0 License (CC BYNC 3.0).

(C) Copyright L. Monserrat et al., 2011

Licensee PAGEPress, Italy

Cardiogenetics 2011; 1:e8

doi:10.4081/cardiogenetics.2011.e8

conduction disorders) and the inherited diseases affecting the aorta (Marfan, Loeys-Dietz, familial thoracic aortic aneurysms). All these entities share some features that we would like to emphasize, as on following.

They are associated with sudden death, especially in the young

The inherited cardiovascular diseases are amongst the most frequent causes of sudden death. ${ }^{9-11}$ In fact, sudden death is frequently the reason for the diagnosis of one of these conditions. Facing a case of sudden death the clinicians have always to consider the cardiomyopathies and the channelopathies. So that, knowledge and expertise in the diagnosis and evaluation of all these conditions are required for those teams, including clinicians and geneticists who have to deal with the problem of sudden death.

They are clinically heterogeneous conditions

Each one of the main inherited cardiovascular diseases we have mentioned could in fact be considered as a collection of multiple different diseases. Morphological and functional manifestations (like the presence of obstruction or the degree and distribution of the hypertrophy in hypertrophic cardiomyopathy), associated extracardiac manifestations (like skeletal myopathy in some cardiomyopathies or hearing loss in long QT syndrome), electrocardiographic abnormalities, age of onset, prognosis, and many other 
characteristics are highly variable in each one of the inherited cardiovascular diseases. This clinical heterogeneity is in part explained by the genetic heterogeneity of the conditions, with multiple different genes and mutations associated with one disease and also multiple diseases associated with the same gene. ${ }^{12,13}$

\section{A familial perspective is essential in their clinical evaluation}

In these diseases the clinician has not to think on a single individual, but in a family. ${ }^{14}$ The diagnosis of an index patient should trigger the clinical screening of the relatives. The main objective of this screening is to identify other members of the family at risk for disease related complications, including sudden death. ${ }^{15}$ But the study of the family is also very useful to achieve the correct diagnosis and to perform the risk stratification of the index patient. Knowledge, training, personnel (cardiologists, nurses, genetic counsellors) and certain infrastructure and organization are required for the correct and cost-efficient evaluation of the families, and once in place may be used to deal with all the inherited cardiovascular conditions. ${ }^{12}$

\section{Genetic diagnosis is feasible in these diseases}

Genetic studies have already been of extraordinary value to progress in the understanding and clinical management of the inherited cardiovascular diseases. As an example, genetics have been the gold standard for the re-evaluation of our diagnostic criteria for relatives in hypertrophic and arrhythmogenic cardiomyopathies. ${ }^{16-18}$ Genetics are also being the clue to recognize and understand the reasons for the presence of overlapping phenotypes. ${ }^{19,20}$ In general the genetic studies are becoming fundamental to understand the clinical heterogeneity and to improve the risk stratification of these diseases. ${ }^{13,21}$ However, and inadequate interpretation of the results of the genetic studies may lead to inappropriate clinical decisions and the use of the genetic studies in patients in which they are not indicated would lead to unnecessary costs. Once we have got the gun (the genetic studies) we find that there are many available targets. However, the bullets are quite expensive and we should not shoot innocent bystanders. Knowledge and training are again required to perform adequate indications and interpretations of the genetic tests.

\section{Clinical interest of the genetic studies in inherited cardiovascular diseases}

The interpretation of the genetic studies

The correct interpretation of the results of the genetic studies requires the evaluation of different scenarios and a stepwise approach summarized in Figure 1. In the following sections we briefly comment the different situations we have to consider when interpreting a genetic result.

A common situation: when we do not identify a genetic variant potentially associated with the disease

In a relevant proportion of cases $(30-40 \%$ in hypertrophic cardiomyopathy, $30-50 \%$ in arrhythmogenic cardiomyopathy or $20-30 \%$ in long QT, for example) we are not able to identify a genetic cause for the disease. ${ }^{13,22}$ One potential explanation is that the clinical diagnosis is wrong, but in many cases the genetic studies are negative even in patients with a definitive clinical diagnosis. There are many other reasons for a negative result, including: i) the disease is not caused by a genetic factor ii) the study is not complete, iii) there are technical limitations and failures in the genotyping, iv) the lack of knowledge about all the genes potentially involved in the origin of the disease, and v) inadequate selection of the candidate genes or of the screening strategy. We comment briefly on each one of these potential reasons:

- the disease may not be secondary to a genetic factor: the differential diagnosis of many inherited cardiovascular diseases frequently includes non-genetic causes. The genetic studies will be negative in a case of suspected cardiomyopathy if the real cause of the phenotype is an adaptation to sport (athlete's heart), in a case of dilated cardiomyopathy secondary to a viral infection, or in patients with QT prolongation secondary to metabolic imbalances or drugs;

incomplete studies: before we assume that the disease is not due to a mutation in any given gene we have to check if the study has included at least all the coding exons and flanking intronic regions of this gene. ${ }^{12}$ Many studies in the literature have included and include only selected fragments of the genes. Some fragments usually present problems for the sequencing. With the standard Sanger sequencing we usually do not study most of the intronic regions. Even though intronic regions are considered as non-coding and are not included in the sequencing there may harbour pathogenic mutations. ${ }^{23,24}$ It is a practical and probably cost-efficient approach not to study most of the intronic regions, but we have to be aware of the limitation originated by this decision. Novel technical approaches like next generation sequencing are not free from these limitations. The results of next generation sequencing studies are dependent on the enrichment process that serves to concentrate the study in the regions of

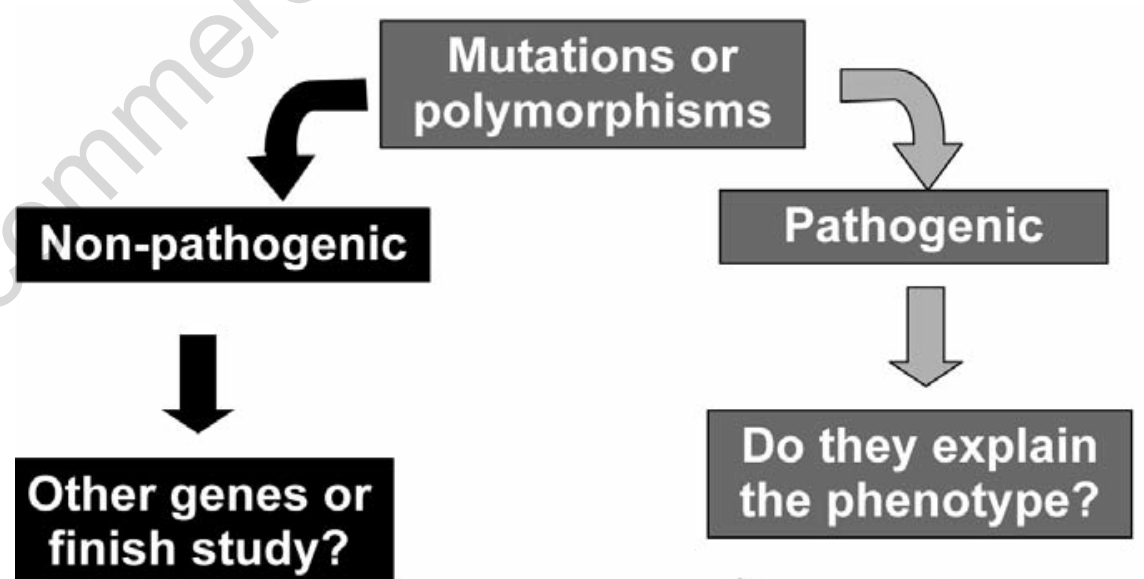

\section{finish study?}
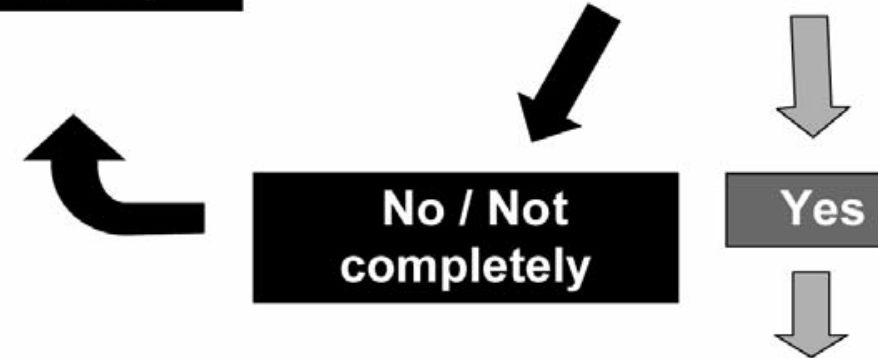

Use available information

Figure 1. Flow chart for the interpretation of the results of the genetic studies in inherited cardiovascular diseases. 
interest (usually exons) and the coverage of these regions of interest is not always complete.

- technical limitations and failures in the genotyping: each of the genotyping techniques has limitations and failures. Sanger sequencing does not allow the identification of some mutations like chromosome rearrangements or big insertion-deletions. ${ }^{25}$ False negative and false positive results are rare, but possible. Next generation sequencing has also false positive and false negative results. All techniques are also dependent on the quality of DNA and failures in the sample tracking, identification, etc. should be considered. Independent double reading of the sequencing results and confirmation of the positive results with a second study in a new PCR product are recommended to reduce the number of false negative and false positive results.

the lack of knowledge about all the genes potentially involved in the origin of the disease: we still do not know all the genes associated with each of the inherited cardiovascular diseases. It is also likely that in a significant number of cases the disease is caused by the interaction of multiple genetic variants in different genes together with environmental factors in a single patient. ${ }^{26}$ These genetic variants could be non-pathogenic when present alone and may be even relatively frequent in the population. Given the enormous number of potential interactions that could be pathogenic it may be extremely difficult to identify the origin of the disease in such cases,

- inadequate selection of the candidate genes or of the screening strategy: if the clinical diagnosis is not correct, or in cases of overlapping phenotypes the orientation of the genetic screening may be erroneous. In that case it could be impossible to find the genetic cause of the disease, because we are studying the wrong genes. Of course this would not be the problem is we are able to study all the genome of the patient, but in that case (still not cost-effective) we would have to face different difficult problems related with the interpretation of the pathogenicity of multiple variants.

\section{The favourable scenario: we identi-}

\section{fy genetic variants that could be}

\section{related with the disease}

When we perform either Sanger or next generation sequencing we compare the results of the genotyping with reference sequences and we usually find multiple genetic variants. To decide whether these variants are or are not associated with the disease is not an easy task. $^{27}$ This is especially true when we study multiple genes and genes that are less well known. This interpretation process could be divided in different steps.

Evaluation of the frequency of the genetic variants in the general population: mutations versus polymorphisms

One of the first steps is to evaluate the frequency of the identified variant(s) in the general population. Those variants that are not rare in the general population are denominated polymorphisms. ${ }^{28}$ There is no exact limit of frequency to define a variant as polymorphism, but we usually use this term for variants present in more than $1 \%$ of the population. ${ }^{12}$ However, when we talk about rare diseases, such as long QT syndrome, a variant present in 1:200 or 1:300 of the healthy individuals could also be considered as a polymorphism. It is relevant to remember that the absence of one variant in a sample of 100 or less controls is not sufficient to establish that the prevalence of this variant in the general population is lower than $1 \%$. We also have to remember that the control individuals should ideally come from the same population as the individual carrying the variant, because different populations and ethnic groups present different polymorphisms. The same variant could be considered a polymorphism in one population but not in others. Polymorphisms are usually not pathogenic, and would not explain rare diseases, however, in some cases variants that are frequent in a given population may be disease causing. ${ }^{29,30}$ As an example, in the Naxos Island the genetic variant responsible for the Naxos disease (a recessive form of arrhythmogenic cardiomyopathy) could be considered as a polymorphism because of its frequency in that particular population, but it is the cause of the disease in homozygous carriers. ${ }^{31}$

We describe as mutations those genetic variants that are very rare in the general population. Even though the term mutation is usually associated with the idea of a deleterious effect, it is very important to remark that not all the mutations are pathogenic. There are harmful or deleterious mutations that are the origin of the monogenic diseases (including the inherited cardiomyopathies), but there are also neutral mutations, with no apparent harmful or beneficial effect, and even advantageous mutations that may provide advantages for the affected individual.

Evaluation of the pathogenicity of the identified genetic variants

For both mutations and polymorphisms we have to decide whether we consider them pathogenic or not. This decision is not easy and in many cases we will not have a definitive con clusion. We have to consider both basic and clinical information (Table 1).

\section{Basic information to define pathogenicity}

With the term basic information we refer to all the data coming from the available knowledge about the molecular implications of the genetic variant. We comment on several of the basic characteristics that should be evaluated:

i) Type of mutation: we can define multiple types of mutation and some of them are more likely to have deleterious consequences. We give some examples:

Silent mutations: in these mutations there is a change in a nucleotide, which doesn't cause a change in the corresponding amino acid. These mutations are usually non-pathogenic, but there are exceptions in cases where the mutation affects regions implicated in the DNA splicing process. ${ }^{32}$

Nonsense mutations are point mutations that cause the introduction of a stop codon that may result in a truncated protein. Missense mutations are those in which a change in one nucleotide results in a change in the resulting amino acid. Both types of point mutations may be pathogenic but usually the effect of nonsense mutations is more severe because they produce more serious alterations on the protein structure. ${ }^{27,33}$

- Frame-shift mutations are caused by nucleotide insertions and or deletions, which alter the reading frame of the sequence and may result in truncated proteins. They are in general more likely to be pathogenic than the missense ones.

ii) Region of the gene and of the protein

Table 1. Basic and clinical information required for the evaluation of the pathogenicity of the genetic variants.

\begin{tabular}{|c|c|}
\hline Basic information & Clinical information \\
\hline Type of mutation & Absence in healthy controls \\
\hline Relevance of the protein affected domains & Cosegretation within families \\
\hline Conservation in different species and isoforms & $\begin{array}{l}\text { Previous descriptions: } \\
\text { - of the same mutation in other individuals }\end{array}$ \\
\hline Bioinformatics studies & - of other mutations in the same amino acid \\
\hline Functional studies & - of other mutations in the same region or domain \\
\hline Animal models & - of other mutations in the same gene \\
\hline
\end{tabular}


affected by the mutation: Those mutations that affect coding regions (exons) are more likely pathogenic than mutations affecting then noncoding intronic regions. Intronic mutations affecting regions next to the exons that are implicated in the splicing process (splice site mutations) are more likely pathogenic than mutations in other intronic regions. However, even point mutations affecting non-flanking regions may be pathogenic (for example creating alternative splice sites). ${ }^{34}$ The effect of the mutations also depends on the functional relevance of the affected region of the protein and mutations in critical points are more likely pathogenic than mutations in regions with less critical functions. ${ }^{32}$

iii) Phylogenetic conservation of the affected amino acids: pathogenicity is more likely when a mutation affects an amino acid which is highly conserved in homologous regions of different species and between different isoforms of the same protein.

iv) Bioinformatics studies: the effect of the same change in amino acid varies depending on the characteristics of the region where it occurs. For missense mutations, the effect of the mutation may also be related with the physicochemical differences between the original and the newly encoded amino acid. The main properties we consider are mass, charge, hydrophobicity and polarity. Different bioinformatics programs provide estimations about the potential of the mutations to produce structural and functional abnormalities in the protein. For mutations affecting flanking intronic regions there are also different programs that provide predictions about the likelihood of an alteration in the splicing process due to those mutations. In any case, all the predictive software gives only indicative information and should not be considered sufficient to either confirm or discard the pathogenicity of a given variant.

v) Functional studies: we include in this group different types of in vitro studies that may be performed to evaluate the functional consequences of the mutations. For example, the expression of mutated channels in oocytes that allows the study of the effect of those mutations in the ion currents; or the evaluation of the velocities of contraction and relaxation in myocardial filaments reconstructed in vitro including mutated proteins. ${ }^{35}$ These studies may clarify the pathogenic potential of the mutations and give also clues about the mechanisms implicated in disease development.

vi) Animal models: the development of transgenic animals expressing a given mutation may provide strong evidence about the pathogenicity of those variants, especially if the animals reproduce the phenotype observed in humans. ${ }^{35,36}$ However, this approach is limited by the cost and difficulties to obtain those models and also because animals and humans have different genes, proteins, physiology, etc. For these reasons the consequences of a mutation in the animal and the human may be completely different.

\section{Clinical information to define pathogenicity}

Basic information may provide good clues about the pathogenicity of a genetic variant, but we could say that it is never definitive. Clinical information is absolutely essential to confirm the pathogenicity of the genetic variant and to define its clinical consequences.

vii) Absence in healthy controls: In general, pathogenic mutations associated with the development of inherited cardiovascular diseases should not be found in healthy controls. We have previously commented on potential exceptions to this rule (recessive mutations).

viii) Cosegregation of the mutation with the disease: Within a given family, the mutation should cosegregate with the disease (the affected members should carry the mutation and non-carriers should not have the disease). When we assess the cosegregation, we have to consider the existence of different inheritance patterns. For example, in autosomal recessive mutations, heterozygous carriers do not present clinical expression of the disease. Even in mutations with autosomal dominant expression, not all the mutant carriers will show disease expression (incomplete penetrance). Young carriers are usually not affected in cardiomyopathies, but it is possible that also some older carriers do not develop the disease. For these reasons, the absence of disease expression in some carriers of a given variant does not exclude its pathogenicity. ${ }^{13}$

Inherited cardiovascular diseases are usually considered monogenic disorders (one geneone mutation-one disease), but in many cases the disease is due to the combination of two or more mutations. In a family with two pathogenic mutations, if we identify only one of the disease causing mutations we may erroneously consider that this mutations does not cosegregate with the disease, because some of the affected members may not have the mutation (they have the other).

ix) Previous descriptions of the mutation: The most valuable information about the pathogenicity and clinical implications of a given mutation will come from the integration of the clinical and genotyping data of multiple families with the same mutation. It has been suggested that most mutations are private (only present in one family) and this would limit the possibility to obtain relevant data from inexistent previous descriptions of the mutation. ${ }^{37}$ In our experience, most of the mutations we find in sarcomeric and ion channels genes have been previously described and clinical information about individuals and families with those mutations provide useful clues about the penetrance, evolution and clinical manifestations of the associated disease. ${ }^{38-40}$ Of course, this is not the situation when we study less well-known genes. There are mutations like $\mathrm{R} 403 \mathrm{Q}^{41}$ or G716R in $\mathrm{MYH} 7^{42,43}$ or $\mathrm{A} 341 \mathrm{~V}$ in $\mathrm{KCNQ} 1^{44}$ that are related to severe phenotypes and high incidence of adverse events; while other mutations are usually associated with mild phenotypes. Mutations like R190W in the LMNA gene are associated with a high incidence of sudden death even in individuals with mild clinical expression of dilated cardiomyopathy. ${ }^{45,46}$ Founder mutations (common origin of the mutation and disease in a significant number of individuals) give the opportunity to define more precisely the phenotype associated with a given mutation. ${ }^{20,47}$ However in those cases we have to consider that the same mutation may have different consequences in patients with a different genetic background.

It is important to remember that the clinical implications and prognosis are very diverse in different mutations affecting the same gene, and even in different mutations affecting the same amino acid. As an example, the phenotype associated with the R403W mutation in $M Y H 7^{48,49}$ is not as severe as the phenotype of R403Q. ${ }^{41}$ We have to consider different levels of detail in the analysis of genotype-phenotype correlations: gene-functional domain-affected residue-specific mutation. Each one of these levels gives us relevant information.

- Gene level: we can identify a general orientation about the clinical expression of mutations in different genes, but there are always multiple exceptions. Some examples: mutations in the $M Y H 7$ gene are usually associated with more severe hypertrophy as compared with mutations in the TNNT2 gene; but not all the genetic variants in the $M Y H 7$ gene are even associated with the development of hypertrophy and there are mutations in the TNNT2 gene associated with severe hypertrophy. Mutations in the TNNT2 may be associated with a high risk of events in the absence of hypertrophy, which is very rare in $M Y H 7$ mutations. Mutations in MYBPC3 gene have in general later disease expression than mutations in other sarcomeric genes, but there are many exceptions to this rule.

- Functional domain level: In a given gene, mutations affecting the same functional domains are likely to have similar functional consequences. For example, in ion channel diseases, pathogenic mutations are usually clustered on amino acids located in pore forming regions and gating domains. LMNA mutations have been associated with multiple phenotypes, including dilated cardiomyopathy with or without conduction system disease, skeletal myopathy (Emery Dreifuss muscular dystrophy, limb-girdle muscular dystrophy) mandibuloacral dysplasia, progeria, etc. and different regions of the protein seem to be 
associated with different phenotypes..$^{50,51}$ As a general observation, mutations affecting amino acids closely located in the protein (not necessarily in the sequence) will have more similar consequences than mutations affecting distant regions.

- Affected residue: Some amino acids may be more susceptible than other to suffer mutations (hot spots) and we usually find different mutations affecting the same amino acid. The presence of other pathogenic mutations in a given amino acid is considered a support to establish the pathogenicity of a novel mutation. Mutations affecting the same amino acid would interfere in the same processes. But even in the same amino acid one variant may be disease causing and others can be well tolerated.

Specific mutation: This is for us the more relevant level of analysis. Each mutation has specific consequences that of course will be modulated by other genetic, epigenetic and environmental factors, but if we collect enough data about each mutation we can establish their general pattern of clinical expression and also we can analyze the specific effects on that mutation of other clinical, environmental and genetic variants. Limitations come from the lack of detailed published information about mutations and their related phenotypes. It is difficult to publish detailed clinical information about mutations, especially if the mutations have been previously described. Registries and databases may be very useful but filling these databases requires time, dedication and knowledge. Clinicians and basic researchers have to be conscious about the potential utility of the detailed information about phenotypes and evolution of their patients and families. In many cases the publications about genetic studies in inherited cardiovascular diseases do not give details on the phenotype of the patients, put together data of patients with multiple different mutations and genes (impossible to evaluate specific characteristics) and/or do not provide data about the relatives (both affected and unaffected, carriers and non-carriers).

According to the previous criteria, the genetic variants will be classified depending on their frequency in the general population (mutations/rare variants/polymorphisms) and their pathogenicity (disease causing/non-disease causing/uncertain pathogenicity). We call pathogenic mutations those variants we are almost sure that are associated with the disease and not present in control individuals. In many cases we will define the mutations as probably associated with the disease or possibly associated with the disease, and finally, in some cases we will define a mutation as not associated with the disease (Figure 2).
Does the pathogenic variant/s explain the phenotype of the patient and the phenotypes of the relatives?

When we find a pathogenic mutation in a patient with an inherited cardiovascular disease we have to consider whether this mutation explain his/her phenotype and the phenotypes of the family. For example, the presence of a mutation usually associated with a mild phenotype and incomplete penetrance may not be enough to explain a severe phenotype. In patients with complex phenotypes (for example left ventricular non-compaction with catecholaminergic polymorphic ventricular tachycardia) we have to consider two possibilities: one mutation may explain both phenotypes, but there could also be two different mutations in the patient. Even if the mutation explains the phenotype of the index patient it may not explain the phenotype of the relatives, as may occur in families with more than one mutation associated with the same or with different phenotypes. If the mutation does not explain the phenotype of the patient or of the family we have to consider the existence of additional mutations either in the same or in different genes. Double or triple mutations are identified in about 5\% of the patients with hypertrophic cardiomyopathy ${ }^{52,53}$ and in more than $10 \%$ of the patients with arrhythmogenic cardiomyopathy. ${ }^{54}$ Double mutations are especially frequent in patients with a severe phenotype $^{52-54}$ The identification of double mutations is crucial for an appropriate genetic counselling and risk stratification in the family. Family history of premature sudden death is a relevant risk factor in all the inherited cardiovascular diseases, however, if the sudden deaths in a family occur in individuals with double mutations, the risk of those relatives carrying only one of the mutations would be lower than the risk expected in the absence of that genetic diagnosis.

Evaluate and use the available information about the consequences of the identified mutation/s

Once we have identified the disease causing mutation or mutations in a family, it is important to collect, analyze and use all the available information about their consequences. It is a common belief that genotype-phenotype correlations are elusive and that the spectrum of clinical expressions in carriers of the same mutations is extremely wide, precluding the possibility to establish of useful genotype-phenotype relationship. We consider that this is a too simplistic approach. In medicine most of the etiologic factors associated with diseases are associated with a variable clinical expression. Infectious agents do not produce the same complications in all the affected patients, but if we study a sufficient number of patients we can establish relevant conclusions about the evolution, prognosis and response to treatment of each agent. Something similar occurs with the genetic causes of inherited cardiovascular diseases, and as we collect more information we learn more about the consequences of the different mutations.

\section{The interpretation of genetic tests in the next generation sequencing era \\ An appropriate interpretation is always fun- damental for the clinical application of genetic studies. This affirmation reaches its maximal}

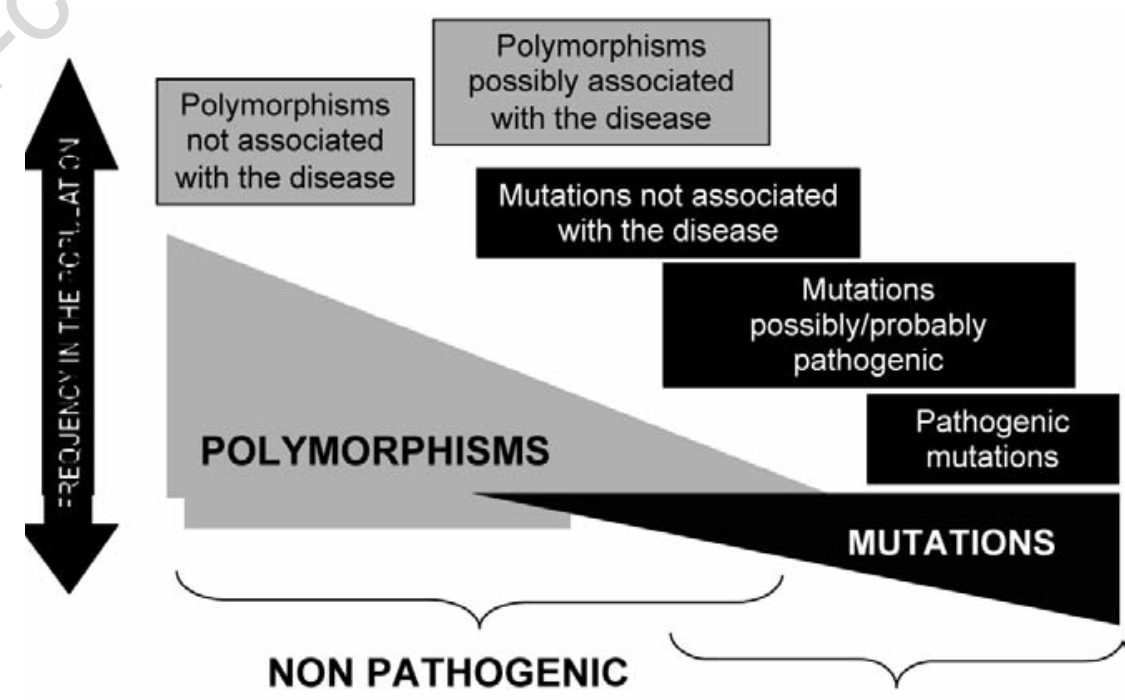

PATHOGENIC

Figure 2. Schematic representation of the different results we can get in a genetic study. We take in consideration the frequency of the identified variants in the general population and the pathogenicity of these variants. Explanation in the text. 
relevance with the introduction of the new genotyping technologies of next generation sequencing. With these genotyping tools we can analyze a big number of genes (even the complete exome or genome) with an assumable cost and in a short time. This is a great advantage both in the clinical and in the research setting. For example we can analyze in a single test all the candidate genes for a given disease, and we can look for novel mutations in genes that have not been previously associated with the disease. Our capacity for identifying genetic variants potentially associated with diseases becomes dramatically increased. But at the same time, we face a parallel increase in the problems for the interpretation of these results. A typical study of a patient with an inherited cardiovascular disease by Sanger sequencing (three to nine genes) may find a maximum of five to ten nonsynonymous genetic variants. A next generation study of 20-40 genes in the same patient would find several hundreds of non-synonymous variants. The study of the complete genome finds more than 2 million variants in a single patient. ${ }^{55}$ of course most of them are common polymorphisms we could discard in our evaluation, but we still face thousands of rare variants in known and unknown genes that either could be associated with multiple different conditions or could be completely innocent. In any test we perform in medicine, the predictive value of the result depends on the pre-test probability (Bayes' theorem), and the probability of any of thousands of variants of unknown significance to be associated with a particular phenotype is very low. In this situation, most of the hypothetical associations we can establish will not be true associations and we will face a very high risk of false positive results. The only way to overcome this problem is to put the focus in the improvement of our skills for the interpretation of the genetic results.

\section{Conclusions}

Genetic studies are clue for the understanding of the heterogeneous clinical manifestations of the inherited cardiovascular diseases. An adequate management of the available information and the collaboration of clinicians and basic researchers in the study of the genotype-phenotype correlations are fundamental to do a proper interpretation of the results and to get the maximal benefit of the genetic tests.

\section{References}

1. Geisterfer-Lowrance AA, Kass S, Tanigawa
G, et al. A molecular basis for familial hypertrophic cardiomyopathy: a beta cardiac myosin heavy chain gene missense mutation. Cell 1990;62:999-1006.

2. Alcalai R, Seidman JG, Seidman CE. Genetic basis of hypertrophic cardiomyoapthy: from bench to the clinics. J Cardiovas Electrophysiol 2008;19:104-10.

3. Curran ME, Splawski I, Timothy KW, et al. A molecular basis for cardiac arrhythmia: HERG mutations cause long QT syndrome. Cell 1995;80:795-803.

4. Priori SG. The fifteen years of discoveries that shaped molecular electrophysiology: time for appraisal. Circ Res 2010;107:4516.

5. Hershberger RE, Siegfried JD. Update 2011: clinical and genetic issues in familial dilated cardiomyopathy. J Am Coll Cardiol 2011;57:1641-9.

6. Ashley EA, Butte AJ, Wheeler MT. Clinical assessment incorporating a personal genome. Lancet 2010;375:1525-35.

7. Wordsworth S, Leal J, Blair E, et al. DNA testing for hypertrophic cardiomyopathy: a cost-effectiveness model. Eur Heart J 2010;31:926-35.

8. Day-Williams AG, Zeggini E. The effect of next-generation sequencing technology on complex trait research. Eur J Clin Invest 2011;41:561-7.

9. Zipes DP, Welens HJ. Sudden cardiac death. Circulation 1998;98:2334-51.

10. Huikuri HV, Castellanos A, Myerburg RJ. Sudden death due to cardiac arrhythmias. N Engl J Med 2001;345:1473-82.

11. Behr ER, Casey A, Sheppard M, et al. Sudden arrhythmic death syndrome: a national survey of sudden unexplained cardiac death. Heart 2007;93:601-5.

12. Tester DJ, Ackerman MJ. Genetic testing for potentially lethal, highly treatable inherited cardiomyopathies/channelopaties in clinical practice. Circulation 2011;123: 1021-37.

13. Charron P, Arad M, Arbustini E, et al. Genetic counselling and testing in cardiomyopathies: a position statement of the European Society of Cardiology Working Group on Myocardial and Pericardial Diseases. Eur Heart J 2010;31:2715-26.

14. Christiaans I, Wilde AA. The patient with hypertrophic cardiomyopathy has a family. Heart 2011;97:262-3.

15. Bai R, Napolitano C, Bloise R, et al. Yield of genetic screening in inherited cardiac channelopathies: how to prioritize access to genetic testing. Circ Arrhythm Electrophysiol 2009;2:6-15.

16. Quarta G, Muir A, Pantazis A, et al. Familial evaluation in arrhythmogenic right ventricular cardiomyopathy: impact of genetics and revised task force criteria. Circulation 2011;123:2701-9.
17. Protonotarios N, Anastasakis A, et al. Arrhythmogenic right ventricular cardiomyopathy/dysplasia on the basis of the revised diagnostic criteria in affected families with desmosomal mutations. Eur Heart J 2011;32:1097-104.

18. Charron P, Dubourg 0, Desnos M, et al. Diagnostic value of electrocardiography and echocardiography for familial hypertrophic cardiomyopathy in genotyped children. Eur Heart J 1998;19:1377-82.

19. Priori SG, Napolitano C, Vicentini A. Inherited arrhythmia syndromes: applying the molecular biology and genetics to the clinical management. J Interv Card Electrophysiol 2003;9:93-101.

20. Monserrat L, Hermida-Prieto M, Fernan$\operatorname{dez} \mathrm{X}$, et al. Mutation in the alpha-cardiac actin gene associated with apical hypertrophic cardiomyopathy, left ventricular non-compaction, and septal defects. Eur Heart J 2007;28:1953-61.

21. Ackerman MJ, Priori SG, Willems S, et al. HRS/EHRA expert consensus statement on the state of genetic testing for the channelopathies and cardiomyopathies. Heart Rhythm Society 2011 Scientific Sessions; May 5, 2011.

22. Fowler SJ, Cerrone M, Napolitano C, Priori SG. Genetic testing for inherited cardiac arrhythmias. Hellenic J Cardiol 2010;51: 92-103.

23. Crotti L, Lewandowska MA, Schwartz PJ, et al. A KCNH2 branch point mutation causing aberrant splicing contributes to an explanation of genotype-negative long QT syndrome. Heart Rhythm 2009;6:212-8.

24. Vatta M. Intronic variants and splicing errors in cardiovascular diseases. Heart Rhythm 2009;6:219-20.

25. Barc J, Briec F, Schmitt S, et al. Screening for copy number variations in genes associated with the long QT syndrome: clinical relevance. J Am Coll Cardiol 2011;57:40-7.

26. Ashley EA, Butte AJ, Wheeler MT, et al. Clinical assessment incorporating personal genome. Lancet 2010;375:1525-35.

27. Kapplinger JD, Landstrom AP, Salisbury $\mathrm{BA}$, et al. Distinguishing arrhythmogenic right ventricular cardiomyopathy/dysplasia-associated mutations from background genetic noise. J Am Coll Cardiol 2011;57: 2317-27.

28. Nussbaum RL, McInnes RR, Willard HF, editors. Thompson \& Thompson Genetics in Medicine. 6th ed. Philadelphia, PA: WB Saunders; 2001. pp. 51-94.

29. Plant LD, Bowers PN, Liu Q, et al. A common cardiac sodium channel variant associated with sudden infant death in African Americans, SCN5A S1103Y. J Clin Invest 2006;116:430-5.

30. Dhandapany PS, Sadayappan S, Xue Y, et al. A common MYBPC3 (cardiac myosin 
binding protein C) variant associated with cardiomyopathies in South Asia. Nat Genet 2009;41:187-91.

31. McKoy G, Protonotarios N, Crosby A, et al. Identification of a deletion in plakoglobin in arrhythmogenic right ventricular cardiomyopathy with palmoplantar keratoderma and woolly hair (Naxos disease). Lancet 2000;355:2119-24.

32. Awad MM, Dalal D, Tichnell C, et al. Recessive arrhythmogenic right ventricular dysplasia due to novel cryptic splice mutation in PKP2. Hum Mutat 2006;27: 1157.

33. Kapa S, Tester DJ, Salisbury BA, et al. Genetic testing for long-QT syndrome: distinguishing pathogenic mutations from benign variants. Circulation 2009;120: 1752-60.

34. Awad MM, Dalal D, Tichnell C, et al. Recessive arrhythmogenic right ventricular dysplasia due to novel cryptic splice mutation in PKP2. Hum Mutat 2006; 27:1157.

35. Song W, Dyer E, Stuckey DJ, et al. Molecular mechanism of the Glu99lys mutation in cardiac actin (ACTC gene) that causes apical hypertrophy in man and mouse. J Biol Chem 2011 May 26. [Epub ahead of print]

36. Ripoll Vera T, Monserrat Iglesias L, Hermida Prieto M, et al. The R820W mutation in the MYBPC3 gene, associated with hypertrophic cardiomyopathy in cats, causes hypertrophic cardiomyopathy and left ventricular non-compaction in humans. Int J Cardiol 2010;145:405-7.

37. Bowles KR, Bowles NE. Genetics of inherited cardiomyopathies. Expert Rev Cardiovasc Ther 2004;2:683-97.

38. Rodríguez-García MI, Monserrat L, Ortiz $\mathrm{M}$, et al. Screening mutations in myosin binding protein $\mathrm{C} 3$ gene in a cohort of patients with Hypertrophic Cardiomyopathy. BMC Med Genet 2010;11: 67.

39. Gimeno JR, Monserrat L, Pérez-Sánchez I, et al. Hypertrophic cardiomyopathy. A study of the troponin- $T$ gene in 127 Spanish families. Rev Esp Cardiol 2009;62:1473-7.

40. Laredo R, Monserrat L, Hermida-Prieto M, et al. Beta-myosin heavy-chain gene mutations in patients with hypertrophic cardiomyopathy. Rev Esp Cardiol 2006;59: 1008-18.

41. Watkins H, Rosenzweig A, Hwang DS, et al. Characteristics and prognostic implications of myosin missense mutations in familial hypertrophic cardiomyopathy. $\mathrm{N}$ Engl J Med 1992;326:1108-114.

42. Hwang T, Lee WH, Kimura A, et al. Early expression of a malignant phenotype of familial hypertrophic cardiomyopathy associated with a Gly716Arg myosin heavy chain mutation in a Korean family. Am J Cardiol 1998;82:1509-13.

43. Ackerman MJ, VanDriest SL, Ommen SR, et al. Prevalence and age-dependence of malignant mutations in the beta-myosin heavy chain and troponin $\mathrm{T}$ genes in hypertrophic cardiomyopathy: a comprehensive outpatient perspective. J Am Coll Cardiol 2002;39:2042-8.

44. Crotti L, Spazzolini C, Schwartz PJ, et al. The common long-QT syndrome mutation KCNQ1/A341V causes unusually severe clinical manifestations in patients with different ethnic backgrounds: toward a mutation-specific risk stratification. Circulation 2007;116:2366-75.

45. Meune C, Van Berlo JH, Anselme F, et al. Primary prevention of sudden death in patients with lamin $\mathrm{A} / \mathrm{C}$ gene mutations. $\mathrm{N}$ Engl J Med 2006;354:209-10.

46. Fernández X, Dumont CA, Monserrat L, et al. Sudden death in a patient with lamin $\mathrm{A} / \mathrm{C}$ gene mutation and near normal left ventricular systolic function. Int J Cardiol 2008;126:136-7.

47. Oliva-Sandoval MJ, Ruiz-Espejo F, Monserrat L, et al. Insights into genotypephenotype correlation in hypertrophic car- diomyopathy. Findings from 18 Spanish families with a single mutation in MYBPC3. Heart 2010;96:1980-4.

48. Tesson F, Dufour C, Moolman JC, et al. The influence of the angiotensin I converting enzyme genotype in familial hypertrophic cardiomyopathy varies with the disease gene mutation. J Mol Cell Cardiol 1997;29: 831-8.

49. Moolman-Smook JC, De Lange WJ, Bruwer $\mathrm{EC}$, et al. The origins of hypertrophic cardiomyopathy-causing mutations in two South African subpopulations: a unique profile of both independent and founder events. Am J Hum Genet 1999;65:1308-20.

50. Hermida-Prieto M, Monserrat L, CastroBeiras A, et al. Familial dilated cardiomyopathy and isolated left ventricular noncompaction associated with lamin $\mathrm{A} / \mathrm{C}$ gene mutations. Am J Cardiol 2004;94:504.

51. Hegele R. LMNA mutation position predicts organ system involvement in laminopathies. Clin Genet 2005;68:31-4.

52. Girolami F, Ho CY, Semsarian C, et al. Clinical features and outcome of hypertrophic cardiomyopathy associated with triple sarcomere protein gene mutations. J Am Coll Cardiol 2010;55:1444-53.

53. Girolami F, Olivotto I, Passerini I, et al. A molecular screening strategy based on beta-myosin heavy chain, cardiac myosin binding protein $\mathrm{C}$ and troponin $\mathrm{T}$ genes in Italian patients with hypertrophic cardiomyopathy. J Cardiovasc Med (Hagerstown) 2006;7:601-7.

54. Xu T, Yang Z, Vatta M, et al. Compound and digenic heterozygosity contributes to arrhythmogenic right ventricular cardiomyopathy. J Am Coll Cardiol 2010;55: 587-97.

55. Ashley EA, Butte AJ, Wheeler MT, et al. Clinical assessment incorporating a personal genome. Lancet 2010;375:1525-35. 